\title{
Setting the Pace for Retinal Development: Environmental Enrichment Acts Through Insulin-Like Growth Factor 1 and Brain-Derived Neurotrophic Factor
}

\author{
Silvia Landi, ${ }^{1,2}$ Francesca Ciucci, ${ }^{1}$ Lamberto Maffei, ${ }^{1,4}$ Nicoletta Berardi, ${ }^{3,4}$ and Maria Cristina Cenni ${ }^{4}$ \\ ${ }^{1}$ Laboratorio di Neurobiologia, Scuola Normale Superiore, I-56100 Pisa, Italy, ${ }^{2}$ National Enterprise for nanoScience and nanoTechnology, Scuola Normale \\ Superiore and Consiglio Nazionale delle Ricerche (CNR)-Istituto Nazionale di Fisica della Materia, I-56100 Pisa, Italy, ${ }^{3}$ Department of Psychology, Florence \\ University, 50121 Florence, Italy, and ${ }^{4}$ Institute of Neuroscience CNR, I-56100 Pisa, Italy
}

\begin{abstract}
Environmental enrichment strongly affects visual system maturation both at retinal and cortical levels. Which molecular pathways are activated by an enriched environment (EE) to regulate visual system development has not been clarified. Here, we show that early [postnatal day 1 (P1) to P7] insulin-like growth factor 1 (IGF-1) injections in the eyes of non-EE rat pups mimic EE effects both in increasing BDNF levels in the retinal ganglion cell layer at $\mathrm{P} 10$ and in determining a more adult-like retinal acuity, assessed with pattern electroretinogram at P25. Blocking IGF-1 action in EE animals during the same early postnatal time window by injecting the IGF-1 receptor antagonist JB1 prevents EE effects both on BDNF expression and on retinal acuity maturation. Reducing BDNF expression in the retina of IGF-1-treated rats prevents IGF-1 effects on retinal acuity development. Finally, we show that tyrosine hydroxylase (TH) expression is increased in the retina of P10 EE and IGF-1-treated rats and that blocking TH expression in EE animals prevents EE from affecting retinal acuity development. Thus, early levels of IGF-1 play a key role in mediating EE effects on retinal development through an action that requires BDNF and involves dopaminergic amacrine cell network.
\end{abstract}

\section{Introduction}

Exposure to an enriched environment (EE) strongly affects visual system maturation. Recent studies suggest that the development and plasticity of the visual cortex and the development of retinal circuitry are controlled by the richness of the environment through molecular factors such as insulin-like growth factor 1 (IGF-1) and BDNF (Cancedda et al., 2004; Ciucci et al., 2007; Landi et al., 2007a,b; Sale et al., 2007). Indeed, blocking retinal BDNF by means of antisense oligonucleotides blocks the effects of exposure to EE on retinal ganglion cell (RGC) dendritic segregation (Landi et al., 2007b) and retinal acuity development (Landi et al., 2007a); blocking IGF-1 in the visual cortex blocks EE effects on visual acuity development (Ciucci et al., 2007). IGF-1 is also a crucial factor mediating the effect of prenatal exposure to EE on the process of programmed RGC death (Sale et al., 2007), which occurs mostly within the first postnatal days. Although these studies begin to unravel the molecular mechanisms underlying the accelerated maturation of visual structures in animals exposed to enriched living conditions, which molecular pathways are activated by EE to regulate visual system development remains to be clarified. In particular, whether IGF-1 con-

Received April 19, 2009; revised July 7, 2009; accepted July 14, 2009.

This work was supported by MIUR PRIN to L.M. We thank Adriano Tacchi, Giulio Cesare Cappagli, and Carlo Orsini for helpful technical assistance. We are very grateful to Dr. Ignacio Torres-Aleman for the gift of the anti-IGF-1 antibody.

Correspondence should be addressed to Silvia Landi, Scuola Normale Superiore-National Enterprise for nanoScience and nanoTechnology, Piazza San Silvestro, 12, I-56100 Pisa, Italy. E-mail: silvia.landi@sns.it.

DOI:10.1523/JNEUROSCI.1857-09.2009

Copyright $\odot 2009$ Society for Neuroscience $\quad 0270-6474 / 09 / 2910809-11 \$ 15.00 / 0$ trols also later stages of retinal development, whether IGF-1 and BDNF act separately or not, and through which mechanisms they act on retinal development is not known.

In the present study, we show that the early increase in retinal IGF-1 found in EE animals is a crucial mediator of EE action on retinal development. It is necessary for EE effects on retinal BDNF expression and on the much later occurring development of retinal acuity, since blocking IGF-1 action in EE animals blocks both effects; in contrast, determining an early increase in retinal IGF-1 by means of intraocular injections of IGF-1 in non-EE animals is sufficient to reproduce both EE effects.

Blocking BDNF expression in the retina of IGF-1-treated animals by means of antisense oligonucleotides prevents IGF-1 from affecting retinal acuity development, suggesting that IGF-1, in its role of mediator of EE effects on retinal acuity maturation, requires the increase in BDNF levels in RGC layer of EE pups.

The dopaminergic amacrine cell network could be the ideal target for EE and IGF-1 action in promoting retinal acuity development. Dopaminergic amacrine cells express BDNF receptor TrkB and are sensitive to BDNF (Cellerino and Kohler, 1997; Cellerino et al., 1998; Lee et al., 2005; Liu et al., 2007; Loeliger et al., 2008). Dopamine levels in the retina affect RGC receptive fields (for review, see Witkovsky, 2004), possibly contributing to retinal spatial resolution. We have tested this hypothesis analyzing the expression of the dopamine key biosynthetic enzyme tyrosine hydroxylase $(\mathrm{TH})$ in EE and IGF-1-treated animals and assessing the efficacy of EE in controlling retinal development in animals with reduced $\mathrm{TH}$ expression. We found that the number of $\mathrm{TH}$-immunoreactive neurons is higher in the retina of EE or 
IGF-1-treated rats at postnatal day 10 (P10), when BDNF expression is enhanced in $\mathrm{EE}$ animals. Blocking $\mathrm{TH}$ expression in the retina of $\mathrm{EE}$ rats during this time window by means of antisense oligonucleotides counteracts EE effects on retinal acuity development.

\section{Materials and Methods}

Animal treatment. All experiments were performed on rats in accordance with the Italian Ministry of Public Health guidelines for the care and use of laboratory animals. Long-Evans hooded rats lived in an animal house with a temperature of $21^{\circ} \mathrm{C}$, a $12 \mathrm{~h} \mathrm{light/dark} \mathrm{cycle,} \mathrm{and} \mathrm{food}$ and water available ad libitum. For both housing conditions, mating were made inside the standard cage.

Rearing environments. In the EE, $7 \mathrm{~d}$ before estimated delivery, pregnant females were transferred to a large wire netting cage $(60 \times 50 \times 80$ $\mathrm{cm}$ ) with three floors containing several foodhoppers, two running wheels (one bigger for adults, the other for postweaning pups) to improve physical activity, and differently shaped objects (tunnels, shelters, stairs) that were completely substituted with others once per week. At least two to three pregnant mothers were housed into the enriched cage, together with four to five filler females.

The standard environment (non-EE) was a standard laboratory cage $(30 \times 40 \times 20 \mathrm{~cm})$ housing one dam with her pups as established by the Italian law for the care of laboratory animals.

After birth, all the litters were housed with their dam until the date of experiment.

Intraocular injections of IGF-1 or JB1. For the analysis of the role of IGF-1 in retinal acuity development, non-EE rats $(n=5)$ received intraocular injections of human IGF-1 (IU100; IBT) (Humbel, 1990; Stewart and Rotwein, 1996), whereas EE rats $(n=5)$ received intraocular injections of the IGF-1 antagonist JB1 (H-1356.0001; Bachem). As a control, the other eye was injected with saline $(0.9 \% \mathrm{NaCl}$; same volume as for IGF-1 or JB1). Injections were performed under ether anesthesia at P1, P4, and P7. IGF-1 concentration in the injected solution was changed between P1 and P7 according to the increasing size of the eye globe to maintain a final intraocular concentration of the factor equal to 100 $\mathrm{ng} / \mu \mathrm{l}$ (Sale et al., 2007). Analogously, JB1 concentration was varied according to the increasing size of the eye globe to maintain a final intraocular concentration of the factor equal to $1 \mathrm{ng} / \mu \mathrm{l}$, demonstrated to be effective for at least $3 \mathrm{~d}$ in vitro (Elmlinger et al., 1998; Pozios et al., 2001; Etgen and Acosta-Martinez, 2003). The volume injected was 250, 500, and $750 \mathrm{nl}$ at P1, P4, and P7, respectively, both for IGF-1 and JB1. Intraocular injections were performed by means of a glass micropipette inserted at the ora serrata and connected to a Hamilton syringe. At P25$\mathrm{P} 26$, pattern electroretinogram (P-ERG) recordings were made, and retinal acuity was determined for the treated and the control eye.

Subcutaneous injections of IGF-1. IGF-1 (IU100; $1.8 \mu \mathrm{g} / \mathrm{g}$ body weight; IBT) (Humbel, 1990; Stewart and Rotwein, 1996; Woodall et al., 1999; Thongsong et al., 2002) was administered via subcutaneous injections in non-EE rats $(n=9)$. Injections were performed daily, under ether anesthesia, for 1 week between P1 and P7. The volume injected was $1 \mu \mathrm{l}$ at P1 and increased according the increase in pup weight. Subcutaneous injections were performed using a catheter with a 20 gauge needle connected to a Hamilton syringe.

Electrophysiological assessment of retinal acuity. P-ERG was recorded in 21 rats [non-EE rats subjected to intraocular injections of IGF-1 in one eye and of saline in the other eye $(n=5)$; EE rats subjected to intraocular injections of JB1 in one eye and of saline in the other eye $(n=5)$; IGF-1 subcutaneously treated animals injected in one eye with BDNF antisense (BDNF-AS) and in the other eye with sense oligonucleotides $(n=5)$; EE rats treated in one eye with $\mathrm{TH}$ antisense (TH-AS) and in the other eye with TH mismatch (TH-MS) oligonucleotides $(n=6)$ ].

Rats were anesthetized with an intraperitoneal injection of $20 \%$ urethane $(0.7 \mathrm{ml} / \mathrm{Hg}$; Sigma). P-ERG was recorded as in the studies by Berardi et al. (1990) and Domenici et al. (1991). The stereotaxic apparatus was oriented with an angle of $\sim 40^{\circ}$ with respect to the position of the screen; P-ERG electrodes were small silver rings positioned on the corneal surface by means of a microelectrode drive, so as to avoid occlusion of the pupil. Visual stimuli were sinusoidal gratings alternated in phase with a fixed temporal frequency of $4 \mathrm{~Hz}$. Recorded signals were filtered $(0.1-100 \mathrm{~Hz})$ and amplified in a conventional manner, computer averaged, and analyzed; 15 packets of 20 sums each (300 events) were averaged for each stimulus spatial frequency, changing randomly the spatial frequency from one record to another. For each spatial frequency, the amplitude of the P-ERG signal was taken as the amplitude of the second harmonic in the averaged signal, calculated by a fast Fourier fransform; the P-ERG amplitude decreases with increasing spatial frequency (Berardi et al., 1990; Rossi et al., 2001). The noise level was estimated by measuring the amplitude of the second harmonic in records in which the stimulus was a blank field. Retinal acuity was taken as the highest spatial frequency still evoking a response above noise level. We have controlled, for every animal, that the pupil remained constricted, as normally is in anesthetized animals during the entire duration of the P-ERG recording. The transparency of the optic media was also checked in every animal throughout the experiments by means of an ophthalmoscope; only the results for animals with clear optics are included in this study.

Preparation of retinal sections for immunohistochemistry. Animals were deeply anesthetized with chloral hydrate and perfused transcardially with $1 \times$ PBS followed by fixative ( $4 \%$ paraformaldehyde, $0.1 \mathrm{~m}$ phosphate buffer, $\mathrm{pH}$ 7.4). Eyes were gently removed, postfixed in $4 \%$ paraformaldehyde in $0.1 \mathrm{~m}$ phosphate buffer, $\mathrm{pH} 7.4$, and cryoprotected in $30 \%$ sucrose. Vertical retinal sections (20 or $16 \mu \mathrm{m}$ thick) were cut using a cryostat and processed for IGF-1, IGF-1 receptor, or BDNF immunohistochemistry as described below.

IGF-1 and IGF-1 receptor immunohistochemistry. The total number of animals used for IGF-1 immunoreactivity analysis was 30: four non-EE and four EE rats at P1, four non-EE and four EE rats at P6, four non-EE rats and four IGF-1 treated non-EE rats at P8, and three non-EE and three EE rats at $\mathrm{P} 9$. The total number of animals used for IGF-1 receptor immunoreactivity analysis was 16: four non-EE and four EE rats at P6 and four non-EE and four EE rats at P8. Retinal sections were permeabilized in $0.3 \%$ Triton X-100 and incubated in 1:50 rabbit polyclonal antiIGF-1 receptor antibody (C-20; sc-713; Santa Cruz Biotechnology) or 1:500 rabbit polyclonal anti-IGF-1 antibody kindly provided by Dr. I. Torres-Aleman (Cajal Institute of Neurobiology, National Research Council, Madrid, Spain). This IGF-1 antibody already has been used for IGF-1 immunohistochemistry by Carro et al. (2000) and Trejo et al. (2001). It has a specificity $<1 \%$ cross-reactivity with either insulin or IGF-II, as determined by competition with ${ }^{125}$ I-IGF-1 (Carro et al., 2000). Also the IGF-1 receptor antagonist known as JB-1 has been shown by the Torres-Aleman group and others to inhibit the action of IGF-1 both in vivo and in vitro (Fernandez et al., 1997). Bound antibodies were detected by incubating sections with biotinylated goat anti-rabbit IgG (1:200; catalog \#BA-1000; Vector Laboratories) followed by fluoresceinconjugated extravidin (1:300; E2761; Sigma). Immunostaining of IGF-1 retinal sections was performed in parallel within the same experimental set. Images of RGC regions were acquired at $20 \times$ magnification using a Zeiss HR Axiocam video camera connected to a Zeiss Axiophot microscope and digitalized by Axiovision software. To compare different specimens, the time of exposure was optimized at the start and then held constant throughout image acquisition. Collected images were imported to the image analysis system MetaMorph (Universal Imaging) and used to evaluate pixel intensity of cellular immunofluorescence. All image analyses were done blind. IGF-1 and IGF-1 receptor pixel intensity in the RGC layer was measured within the entire RGC layer area. The resulting mean pixel intensity value was divided by average signal density in overlying equivalent regions in the outer nuclear layer (ONL). IGF-1 and IGF-1 receptor immunoreactivity and mRNA are not detectable in the ONL at this early age (Bondy, 1991; Lee et al., 1992; Sale et al., 2007). Normalized pixel intensity values obtained from at least 8-10 retinal fields were used to calculate the average normalized pixel intensity value in the RGC layer per animal (Landi et al., 2007a,b; Sale et al., 2007).

BDNF immunohistochemistry. A total of 14 Long-Evans hooded rats, aged P10, were used (non-EE rats treated with IGF-1, $n=7$; EE treated with JB1, $n=7$ ). After a blocking step, retinal sections were permeabilized in $0.01 \%$ Triton X-100 and incubated in 1:400 antihuman BDNF 
antibody (catalog \#G1641; Promega). Bound antibody was detected by incubating sections with biotin-conjugated anti-chicken IgG (1:200; catalog \#G2891; Promega), followed by fluorescein-conjugated extravidin (1:300; E2761; Sigma). Images of RGC portions were acquired with a confocal Olympus microscope at $20 \times$ magnification [numerical aperture (NA), 0.7; field, $707 \times 707 \mu \mathrm{m}$ acquired at $1024 \times 1024$ pixels]. Settings for laser intensity, gain, offset, and pinhole were optimized initially and held constant through the experiment. Then, the collected images of the retina were imported to the image analysis system MetaMorph (Universal Imaging) and used to evaluate pixel intensity of cellular immunofluorescence. For each animal, at least 24 retinal sections (12 for the IGF-1- or JB1-treated eye and 12 for saline-treated eye) were analyzed. All image analyses were done blind. The profile of single cells in the RGC layer was outlined, and pixel intensity was measured within this area. Normalized BDNF immunoreactivity levels were calculated as the ratio between the pixel intensity of RGC profiles and the background level, measured in the ONL as described by Landi et al (2007a,b).

TH immunohistochemistry. TH immunohistochemistry was performed on whole-mount retinas. A total of 18 retinas from P10 LongEvans hooded rats were used (non-EE rats treated with IGF-1, $n=7$; non-EE rats treated with saline, $n=6$; EE rats, $n=5)$. After the cryoprotection step, eyes were dissected, and the retina was isolated; wholemount retinas were permeabilized in $0.3 \%$ Triton X-100 for $1 \mathrm{~d}$ and incubated for $4 \mathrm{~d}$ in 1:500 TH-AS antibody (catalog \#MAB318; Chemicon). Bound antibody was detected by incubating sections with 1:200 anti-rabbit biotinylated secondary antibody made in goat (BA-1000; Vector Laboratories), followed by CY3-conjugated extravidin (1:500; catalog \#E4142; Sigma). The number of TH-positive cells was counted after a "blind procedure" in the RGC layer of analyzed retinas in fields of $100 \times 100 \mu \mathrm{m}$ at $40 \times$ magnification using a Zeiss computerized microscope with Stereo Investigator software (MicroBrightField). The total number of TH-positive cells per retina was estimated by multiplying the total number of cells sampled per field times the ratio of the total area of each retina to field area. Images from whole-mount retinas were acquired with a confocal Olympus microscope at $20 \times$ magnification (NA, 0.7; field, $707 \times 707$ $\mu \mathrm{m}$ acquired at $1024 \times 1024$ pixels) to show the density of TH-positive cells in retinas of $\mathrm{P} 10 \mathrm{EE}$ rats intraocularly treated with TH-AS or TH-MS oligonucleotides at P6 and P9.

Intraocular injections of oligonucleotides. Non-EE IGF-1-treated animals received intraocular injections of antisense or sense BDNF phosphorothioate oligonucleotides ( $500 \mu \mathrm{M}$; Eurogentec; injection volume at P6, $500 \mathrm{nl}$, and at P9, $1000 \mathrm{nl}$, according to the size of the eye globe; estimated oligo concentration in the eye, $25 \mu \mathrm{M}$ ) (Menna et al., 2003). Injections were performed under ether anesthesia at P6 and P9, which is in correspondence with the period of enhanced BDNF protein levels in EE; recent findings from our laboratory have demonstrated that intraocular injections of BDNF oligonucleotides reduces retinal expression of BDNF in rats during postnatal development (Menna et al., 2003; Mandolesi et al., 2005; Landi et al., 2007a).

Intraocular injections of BDNF sense (BDNF-S) and antisense oligos were performed using a glass micropipette inserted at the ora serrata connected to a Hamilton syringe. Sequences of the BDNF-AS to reduce BDNF mRNA translation and sense oligonucleotides (targeted to the BDNF translation initiation codon) were 5'-CATCACTCTTCTCACCTGGTGGAAC- ${ }^{\prime}$ and 5'-GTTCCACCAGGTGAGAAGAGTGATG3 ', which correspond to nucleotides 51-75 of the BDNF mRNA and are the same used by Menna et al. (2003) and Mandolesi et al. (2005) to effectively reduce BDNF levels in the developing retina. Fully phosphorothioate oligonucleotides were dissolved in saline with stock solutions of $1 \mathrm{~mm}$. Stock solutions were preserved at $--80^{\circ} \mathrm{C}$ and diluted in saline at the desired concentration at the time of the injection. P-ERG recordings were made at P25-P26, and retinal acuity was determined for each oligo-injected non-EE IGF-1 animal as described previously. Five non-EE IGF-1 rats were intraocularly injected with antisense oligos in one eye, whereas the other eye was treated with sense oligos. In these animals, P-ERG recordings from both eyes were made at P25-P26 to compare in the same non-EE IGF-1 animal the retinal acuity of the normal eye $(n=5)$ and of the eye with decreased BDNF levels $(n=4)$.
For the study of the role of the dopamine on the maturation of retinal acuity in EE animals, EE rats received intraocular injections of TH-AS or TH-MS phosphorothioate oligonucleotides (Eurogentec; injection volume at P6, $500 \mathrm{nl}$, and at P9, $750 \mathrm{nl}$, according to the size of the eye globe; estimated oligo concentration in the eye, $0.6 \mu \mathrm{g} / \mu \mathrm{l})$. Injections were performed under ether anesthesia at P6 and P9, which is in correspondence with the period of an enhanced number of TH-positive amacrine cells in EE animals.

Intraocular injections of TH-AS and TH-MS oligos were performed using a glass micropipette inserted at the ora serrata connected to a Hamilton syringe. The 20-base sequence of the TH-AS oligonucleotide to reduce TH mRNA translation (targeted to the $\mathrm{NH}_{2}$ terminus around the initiating codon (Probst and Skutella, 1996) was 5'-GGTGGGCATAGTGCAAGCTG-3', and that of the mismatch oligonucleotide was 5'-GATCCGCATAGGGCAAGCTG-3' as described by Skutella et al. (1997) in the rat substantia nigra. Fully phosphorothioate oligonucleotides were dissolved in saline with stock solutions of $2 \mathrm{~mm}$. Stock solutions were preserved at $-80^{\circ} \mathrm{C}$ and diluted in saline at the desired concentration at the time of the injection.

To document whether intraocular oligonucleotide injections decreased $\mathrm{TH}$ expression also in the retina, we performed immunohistochemistry on whole-mount retinas from untreated $(n=3)$, TH-MS $(n=3)$, and TH-ASinjected $(n=4)$ rats $24 \mathrm{~h}$ after the last injection, and the number of THpositive cells was counted after a blind procedure.

P-ERG recordings were made at P25-P26, and retinal acuity was determined for each oligo-injected EE animal as described previously. Six EE rats were intraocularly injected with antisense oligos in one eye, whereas the other eye was treated with mismatch to control for the aspecific effects of oligo injections. In these animals, P-ERG recordings from both eyes were made at P25-P26 to compare in the same EE animals the retinal acuity of the normal eye $(n=5)$ and of the eye with decreased TH levels $(n=6)$.

Statistics. We have analyzed our data using the statistical software SigmaStat 3.0. To compare two groups, we used the $t$ test or Mann-Whitney rank sum test, according to the results of the test for normality of the data. The $t$ test was used for normally distributed data, and the nonparametric Mann-Whitney rank sum test for non-normally distributed data. To compare many groups, we have used one-way ANOVA or KruskalWallis ANOVA on ranks according to the results of the test for normality of the data; post hoc comparisons were performed with the Tukey's or Dunn test. To compare results obtained for different treatments at different ages, we used two-way ANOVA and the post hoc Holm-Sidak method. To compare the retinal acuity of the two eyes in animals with one eye injected with IGF-1, JB1, BDNF-AS oligonucleotides, or TH-AS oligonucleotides and the other with the control treatment, we used a paired $t$ test or Wilcoxon signed rank test according to the results of the test for the normality of the data. The difference between groups is considered significant if $p<0.05$. The $p$ level resulting from the test is reported in the text or in the figure legends.

\section{Results}

\section{IGF-1 expression is enhanced in the RGC layer of enriched} pup retinas

Recent experiments show that enriched environmental conditions provided to the dam during the complete period of pregnancy modulate retinal IGF-1 expression in the fetuses (Sale et al., 2007). We have examined IGF-1 protein postnatal expression in the retinas of the offspring of pregnant females transferred to an enriched cage a few days before the delivery. We found that normalized IGF-1 immunoreactivity in the RGC layer of EE rats (calculated as the ratio between the pixel intensity in RGC layer and the pixel intensity in the ONL layer) was statistically higher than in non-EE rats at P1 and at P6 (Fig. 1 ) ( $t$ test, $p=0.037$ at P1 and $p=0.019$ at P6) showing that maternal enrichment from the last days of pregnancy is sufficient to determine an early postnatal IGF-1 increase in the retina of EE pups. At P9, IGF-1 expression in EE versus non-EE rats no longer differs, in agreement with the results of Sale et al. (2007) [EE, $1.603 \pm 0.102$ vs non-EE, $1.620 \pm$ 
0.191; $p=0.942$, two-way ANOVA; age $(\mathrm{P} 1, \mathrm{P} 6$, and P9) $\times$ housing condition (non-EE or EE): factor age, significant, $p<0.001$; factor housing condition, significant, $p=0.009$; interaction, $p=0.040$; post hoc Holm-Sidak method].

\section{IGF-1 mediates the effects of EE on retinal acuity development}

To investigate whether the early increase in retinal IGF-1 in EE animals is a first crucial event to set in motion the process leading to the acceleration of retinal acuity maturation caused by EE (Landi et al., 2007a), we assessed whether an increase in IGF-1 levels in the retina of non-EE rats, achieved by infusing IGF-1 in the eye, was sufficient to mimic EE effects on retinal acuity development. We chose to inject non-EE pups from P1 to P7 because the marked increase in IGF-1 expression found in the retinas of EE with respect to non-EE pups is present in an early time window after birth (P1-P6) (Fig. 1) and no longer present around $\mathrm{P} 10$, as seen by us and by Sale et al. (2007). Analysis of IGF-1 receptor presence in the retina of $\mathrm{EE}$ and non-EE pups at P6 and P8, the same period during which IGF-1 injections were made in non-EE pups, revealed that IGF-1 receptor immunoreactivity is clearly detectable in the RGC layer (supplemental Fig. 1, available at www.jneurosci. org as supplemental material). The presence of IGF-1 receptors at such an early age is in line with previous papers showing that IGF-1 receptors are present very early in the retina (Rodrigues et al., 1988; Waldbillig et al., 1988; Lee et al., 1992; Lofquist et al., 2009) and, in particular, in the rat retina, already at P0 (Lee et al., 1992). At P0, they are expressed in the RGC layer and in the optic nerve fiber layer (Lee et al., 1992).

We injected rats every $3 \mathrm{~d}$ with IGF-1 in one eye and with saline in the other eye as control. We recorded retinal acuity by P-ERG at P25 both for the IGF-1-treated and the saline-treated eye. P25 is the age at which retinal acuity in EE animals becomes higher than in non-EE ones (Landi et al., 2007a). We found that retinal acuity for the IGF-1-treated eyes was significantly higher than for the saline-treated eyes or for the eyes of untreated animals (Fig. 2B); the latter two do not differ; the effects of IGF-1 treatment are comparable with those produced by EE: retinal acuity of IGF-1-treated eyes does not differ from retinal acuity of P25 EE animals (one-way ANOVA and post hoc Tukey's test; IGF-1-treated eyes vs saline-treated eyes, $p<0.001$; IGF-1-treated eyes vs non-EE eyes, $p<0.001$; IGF-1-treated eyes vs EE untreated eyes, $p=0.993)$. In three animals, it was possible to determine retinal acuity for both eyes (Fig. 2C); it is evident
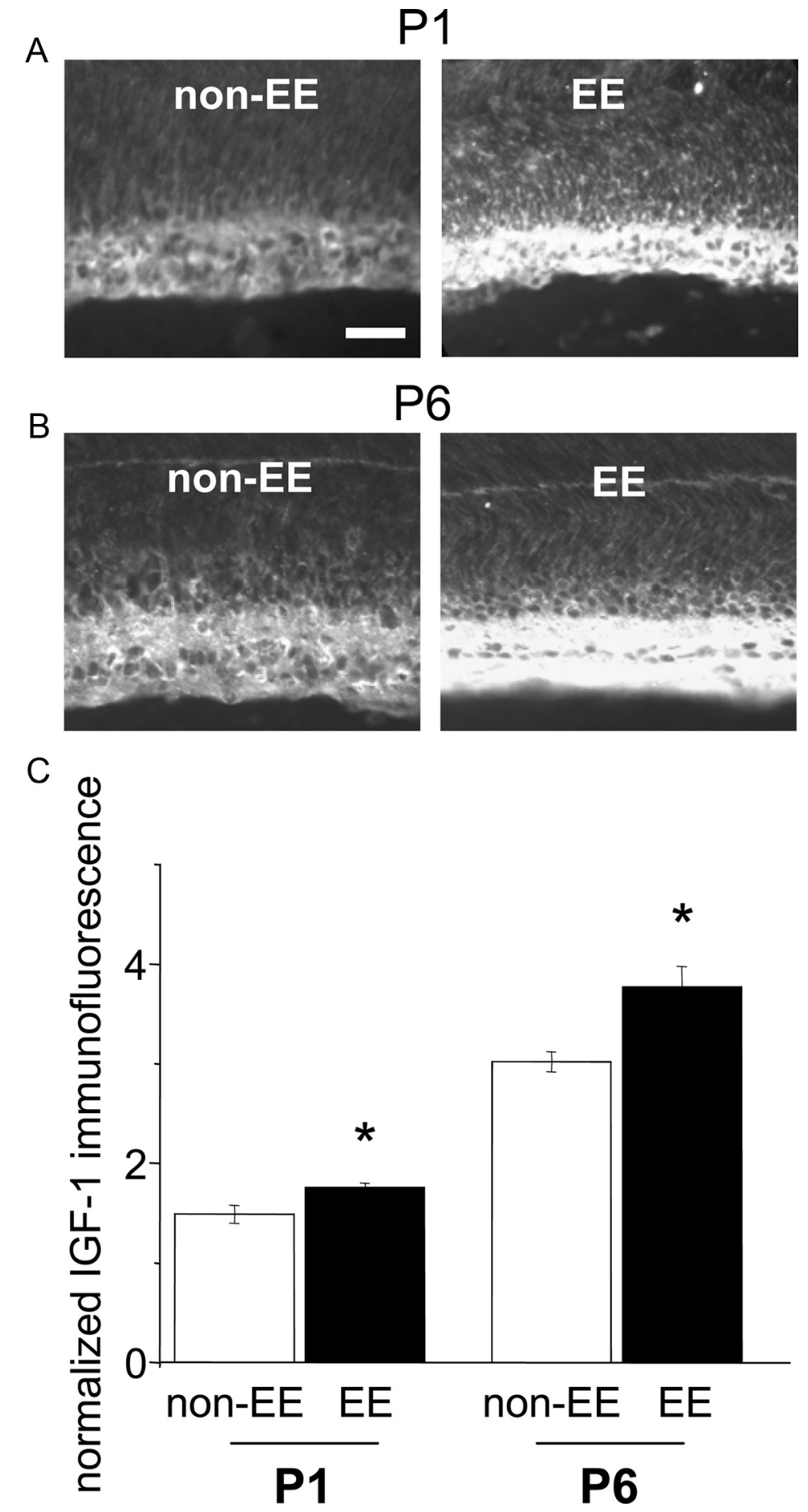

Figure 1. IGF-1 expression is enhanced in the RGC layer of EE pup retinas. Pregnant females were exposed to EE or non-EE, and the pups were examined at P1 and P6 for IGF-1 immunoreactivity. $\boldsymbol{A}, \boldsymbol{B}$, Representative images of P1 and P6 non-EE and EE pup retinas. Scale bar, $50 \mu \mathrm{m}$. C, Quantitative analysis of mean IGF-1 immunofluorescence intensity in the RGC layer normalized to mean background level in the ONL layer. Astrisks denote that the difference between normalized IGF-1 immunoreactivity in the RGC layer of EE and non-EE rats is statistically significant (P1, EE, $1.76 \pm 0.04$ vs non-EE, $1.49 \pm 0.09 ; t$ test, $p=$ $0.037 ; \mathrm{P} 6, \mathrm{EE}, 3.77 \pm 0.21$ vs non-EE, $3.02 \pm 0.1 ; t$ test, $p=0.019$ ). Error bars represent SEM.

that for all three animals, the acuity of the IGF-1-treated eye is higher than that for the contralateral eye (paired $t$ test, $p=0.018$ ).

To assess whether the early retinal IGF-1 increase is necessary for EE to affect retinal acuity development, we antagonized IGF-1 action in EE rats. IGF-1 action in the retina was antagonized injecting EE rats at $\mathrm{P} 1, \mathrm{P} 4$, and $\mathrm{P} 7$ with JB1, an IGF-1 receptor antagonist (Fernandez et al., 1997; Carro et al., 2000; Ciucci et al., 
A intraocular injections of IGF-1 or JB1

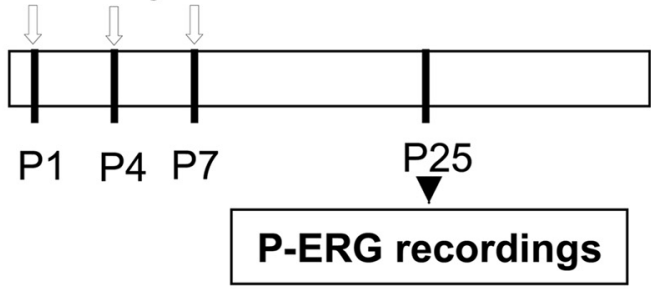

$\mathrm{B}$

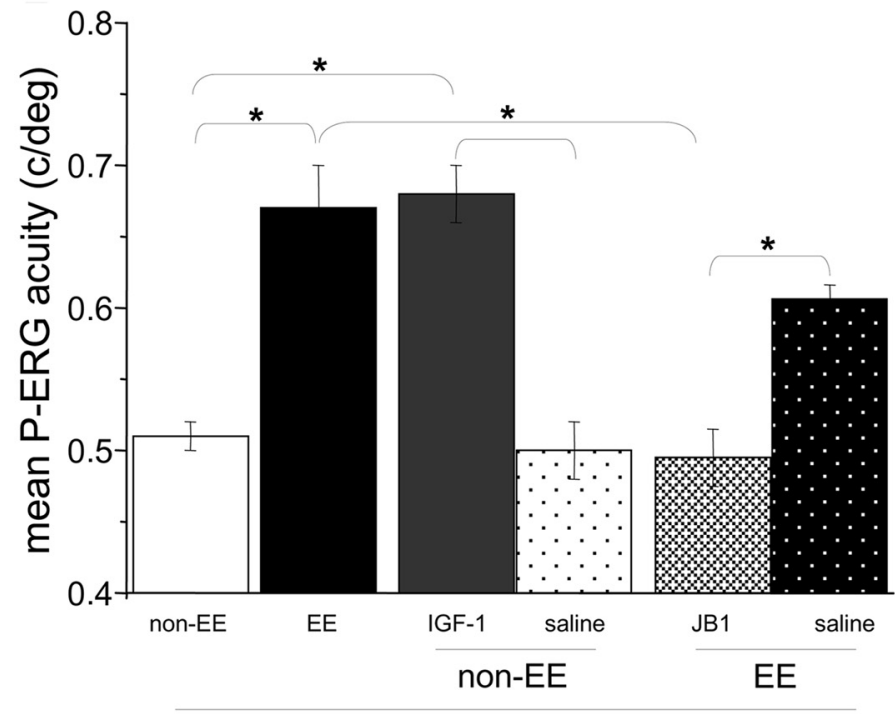

P25

$\mathrm{C}$
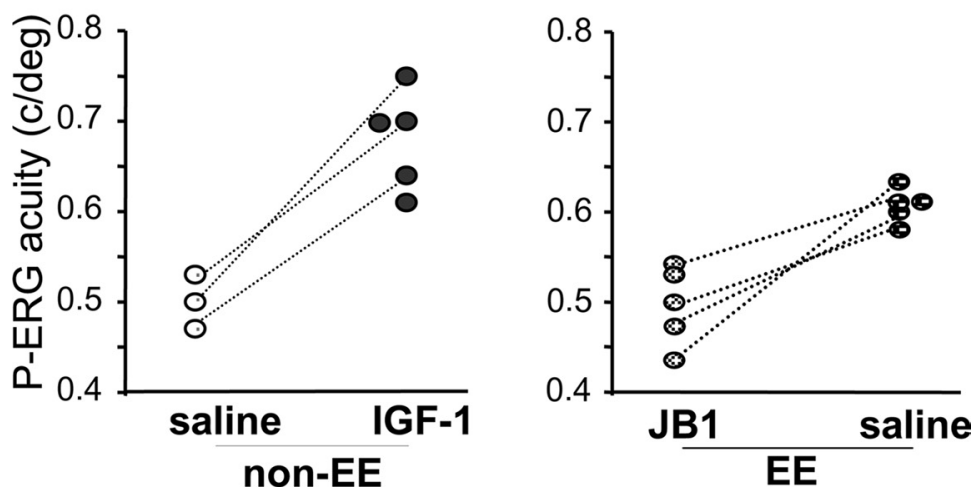

Figure 2. IGF-1 intraocular injections affect retinal acuity development in standard reared rats, whereas IGF-1 blockade prevents EE effects on retinal acuity development in enriched animals. $\boldsymbol{A}$, Schematic protocol of the experiment. $\boldsymbol{B}$, Mean P-ERG acuity assessed at P25 for non-EE animals intraocularly treated with IGF-1 or saline and EE animals intraocularly treated with JB1 or saline. Mean P-ERG acuity in P25 non-EE $[0.51 \pm 0.01$ cycles/degree $(\mathrm{c} / \mathrm{deg}) ; n=5]$ and EE $(0.67 \pm 0.03 \mathrm{c} / \mathrm{deg} ; n=5)$ animals is reported for comparison. Retinal acuity in IGF-1-treated eyes of non-EE animals (non-EE IGF-1, $0.68 \pm 0.02 \mathrm{c} / \mathrm{deg} ; n=5$ ) is significantly higher (asterisks) than in non-EE saline-treated eyes (non-EE saline, $0.5 \pm 0.02 \mathrm{c} / \mathrm{deg} ; n=3$ ) and in non-EE untreated animals, but it is comparable to that reached in EE animals (one-way ANOVA, $p<0.001$, posthoc Tukey's test; non-EEIGF-1 vs non-EE saline, $p<0.001$; non-EEIGF-1 vs non-EE, $p<0.001$; non-EE IGF-1 vs EE, $p=0.993$ ). Mean retinal acuity at $\mathrm{P} 25$ for the eyes of EE animals intraocularly injected with JB1 (EE JB1; $n=5$ ) or with saline (EE saline; $n=5$ ) is shown. P-ERG acuity of EE JB1-treated animals is significantly lower (asterisks) than in EE animals either treated with saline or untreated and does not differ from the P-ERG acuity of P25 non-EE animals (one-way ANOVA, $p<0.001$, post hoc Tukey's test; $\mathrm{EE}$ vs $\mathrm{EEJ} \mathrm{JB} 1, p<0.001$; EE saline vs EE JB1, $p=0.003$; EE JB1 vs non-EE, $p=0.963$; EE vs EE saline, $p=0.079$ ). Error bars represent SEM. C, Left, Data for single non-EE animals injected in one eye with IGF-1 (non-EE IGF-1; $n=5$ ) and in the contralateral eye with saline (non-EE saline; $n=3$ ). In three animals, retinal acuity was determined for both eyes (data points joined by dotted lines); $i$ i is evident that for all three animals, the acuity of the IGF-1-treated eye is higher than that for the contralateral eye (paired $t$ test, $p=0.018$ ). Right, Data for single EE animals injected in one eye with JB1 (EEJB1; $n=5$ ) and in the contralateral eye with saline (EE saline; $n=5$ ). In four animals, retinal acuity was determined for both eyes (data points joined by dotted lines); it is evident that for all four animals, the acuity of the JB1-treated eye is lower than that for the contralateral eye (paired $t$ test,$p=0.024$ ).
2007) in one eye, whereas the other eye was injected with saline as the control. Retinal acuity was assessed at $\mathrm{P} 25$ for both eyes. We found that JB1 treatment blocked EE effects on retinal acuity maturation (Fig. 2B). P-ERG acuity assessed for the JB1-treated eyes of EE rats was significantly lower than the retinal acuity found for the eyes treated with saline or for the eyes of EE untreated animals and did not differ from the retinal acuity of the eyes of P25 non-EE rats ( $p<0.001$, oneway ANOVA and post hoc Tukey's test; EE untreated eyes vs EE JB1-treated eyes, $p<$ 0.001 ; EE saline-treated eyes vs EE JB1treated eyes, $p=0.003$; EE JB1-treated eyes vs non-EE eyes, $p=0.963$; EE $v$ s. EE saline-treated eyes, $p=0.079$ ). In four animals, we have been able to assess retinal acuity for both eyes; it is evident that for all four animals, the acuity of the JB1treated eye is lower than that for the contralateral eye (paired $t$ test, $p=0.024$ ) (Fig. 2C).

These results support the hypothesis that the early increase in retinal IGF- 1 is a crucial event in mediating $\mathrm{EE}$ effects on retinal maturation.

\section{Intraocular IGF-1 administration triggers the enhancement of BDNF expression in RGC layer at P10} Landi et al. (2007a,b) have shown that EE increases BDNF expression in the RGC layer around P10 and that this increase in BDNF expression is necessary for EE effects on the maturation of retinal acuity. Having now shown that IGF-1 intraocular administration from P1 to P7 mimics EE effects on retinal acuity maturation, we asked whether IGF-1 could affect BDNF expression.

We investigated whether IGF-1 treatment affected BDNF expression in the RGC layer by immunohistochemistry. We intraocularly injected non-EE animals at P1, P4, and P7 with IGF-1 in one eye (Fig. $3 A$ ). The other eye was injected with saline as the control. We analyzed BDNF expression at P10. We found that IGF-1 treatment affects BDNF protein expression (Fig. 3B,C); BDNF immunoreactivity is significantly higher in the RGC layer of IGF-1-treated eyes than in the salinetreated ones (Mann-Whitney rank sum test, $p<0.001)$.

IGF-1 blockade prevents BDNF enhanced expression in RGC layer of EE animals at P10

To determine whether the increase in BDNF expression found in EE rats results from the IGF-1 increase caused by EE, we 
injected EE pups with JB1 in one eye and with saline in the other eye. We found that $\mathrm{BDNF}$ protein levels are not affected by EE if IGF- 1 action is antagonized (Fig. 3B,C): BDNF levels in the eyes of JB1-treated EE animals do not differ from those found in non-EE saline-treated ones and are significantly lower than in the saline-treated eyes of EE animals (Kruskal-Wallis oneway ANOVA on ranks, $p<0.001)$. These data show that the early IGF- 1 increase in the retina is both necessary and sufficient to determine an enhancement of BDNF expression in the RGC layer.

\section{Block of BDNF in the retina prevents \\ IGF-1 effects on retinal \\ acuity development}

We already know that blocking BDNF expression in EE animals around P10 blocks EE effects on retinal development (Landi et al., 2007a,b). To assess whether IGF-1 requires BDNF to affect retinal functional development, we antagonized BDNF action in IGF-1-treated rats and assessed whether this blocked IGF-1 action on retinal acuity development. To reduce the number and volume of injections in the eyes, IGF-1 was administered subcutaneously in non-EE rats from P1 to P7 (daily injections) (Fig. 4A). We have controlled that this treatment is effective in increasing IGF-1 retinal levels (Fig. $4 \mathrm{~B}$ ); indeed, IGF-1 levels in the RGC layer are significantly higher at $\mathrm{P} 8$ in IGF-1-treated rats with respect to untreated (control) rats $(t$ test, $p=0.002$ ). We then injected rats subjected to IGF-1 subcutaneous treatment with BDNF-AS oligos in one eye and BDNF-S oligos in the other eye, as described by Landi et al. (2007a). These animals have been recorded at $\mathrm{P} 25$, and the P-ERG acuity of the BDNF-AS-treated eye was compared with that of the BDNF-S -treated eye. We found that retinal acuity of the BDNF-AS-treated eye was significantly lower than that recorded from the eyes of EE untreated animals or BDNF-S-treated animals and does not differ from that of non-EE rats (Fig. 4C) (one-way ANOVA, $p<0.001$; post hoc Tukey's test; BDNF-AS-treated eye vs EE untreated eyes, $p=0.009$; BDNF-AStreated eyes vs BDNF-S treated, $p=0.032$; BDNF-AS-treated eyes vs non-EE untreated eyes, $p=0.572$ ). In four animals, we succeeded in recording retinal acuity from both eyes, the BDNF-AS-treated and the BDNF-S-treated ones; it is evident that the acuity of the antisensetreated eye is lower than that for the contralateral, sense-treated eye (paired $t$ test, $p=0.027$ ) (Fig. 4C).

Thus, the reduction of retinal BDNF blocks the effects of IGF-1 on retinal functional maturation. All together, our results suggest that EE determines an early increase in retinal

A

B

C represent SEM.

\section{Intraocular injections of IGF-1 in non-EE rats or JB1 in EE rats}

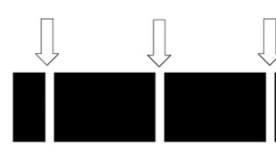

-
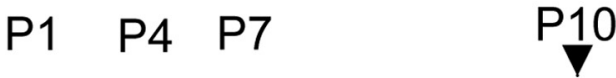

immunoreactivity for BDNF nOn-EE
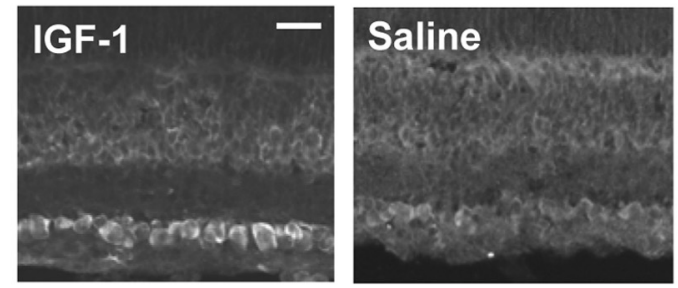

EE
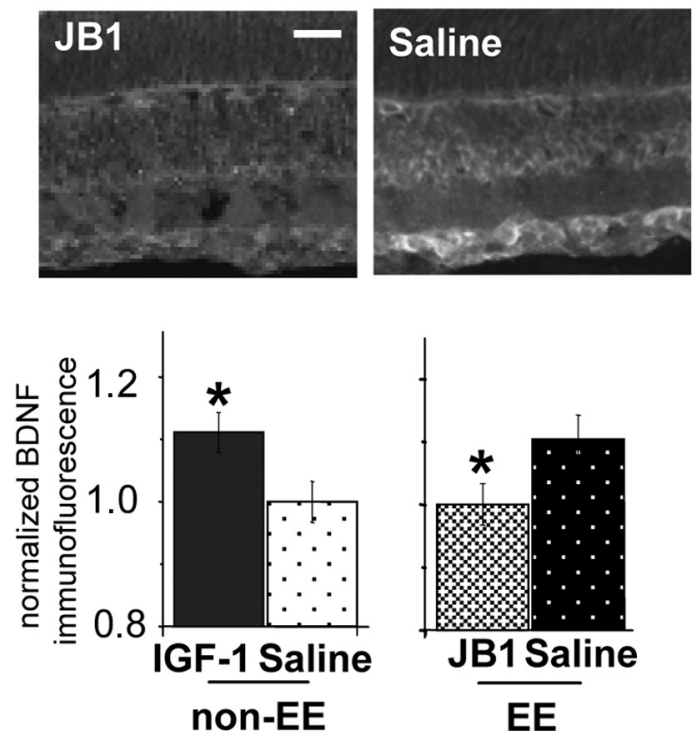

Figure 3. IGF-1 expression controls BDNF protein levels in RGC layer at P10. $\boldsymbol{A}$, Experimental protocol. $\boldsymbol{B}$, Retinal section micrographs of P10 non-EE rats intraocularly injected with IGF-1 or saline (top) and of P10 EE rats intraocularly injected with JB1 or saline (bottom). BDNF-immunolabeled cells are detectable at the level of the RGC layer. Scale bars, $50 \mu \mathrm{m}$. C, Left, Quantitative analysis of mean BDNF immunofluorescence intensity in the RGC layer normalized to mean background level in the ONL layer of non-EE rats intraocularly injected with IGF-1 or saline and immunostained for BDNF at P10. BDNF immunoreactivity is significantly higher (asterisk) in the retinas of non-EE IGF-1-treated rats $(1.103 \pm 0.033 ; n=7)$ with respect to those of non-EE saline-treated rats $(1 \pm 0.033 ; n=7)$ (Mann-Whitney rank sum test; $p<0.001)$. Right, Mean BDNF immunofluorescence intensity in the RGC layer normalized to mean background level in the ONL layer of EE rats intraocularly injected with JB1 or vehicle and immunostained for BDNF at P10. BDNF immunoreactivity is significantly lower (asterisk) in the retinas of EE JB1-treated rats $(1.001 \pm 0.033 ; n=$ 7) with respect to those of EE saline-treated rats $(1.104 \pm 0.039 ; n=5)$ (Mann-Whitney rank sum test; $p<0.001)$. Error bars

IGF-1, which then causes an increase in retinal BDNF, which then determines a more adult-like retinal acuity at P25.

Role of dopaminergic amacrine cells in retinal maturation in EE animals

In the rat retina, dopaminergic amacrine cells express TrkB (Cellerino and Kohler, 1997); their development is accelerated by BDNF intraocular injections from P8 to P14 (Cellerino et al., 
A

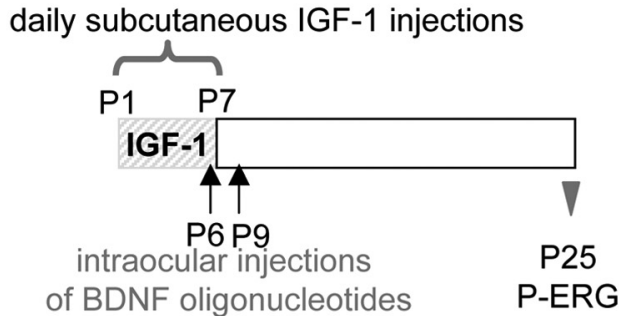

B
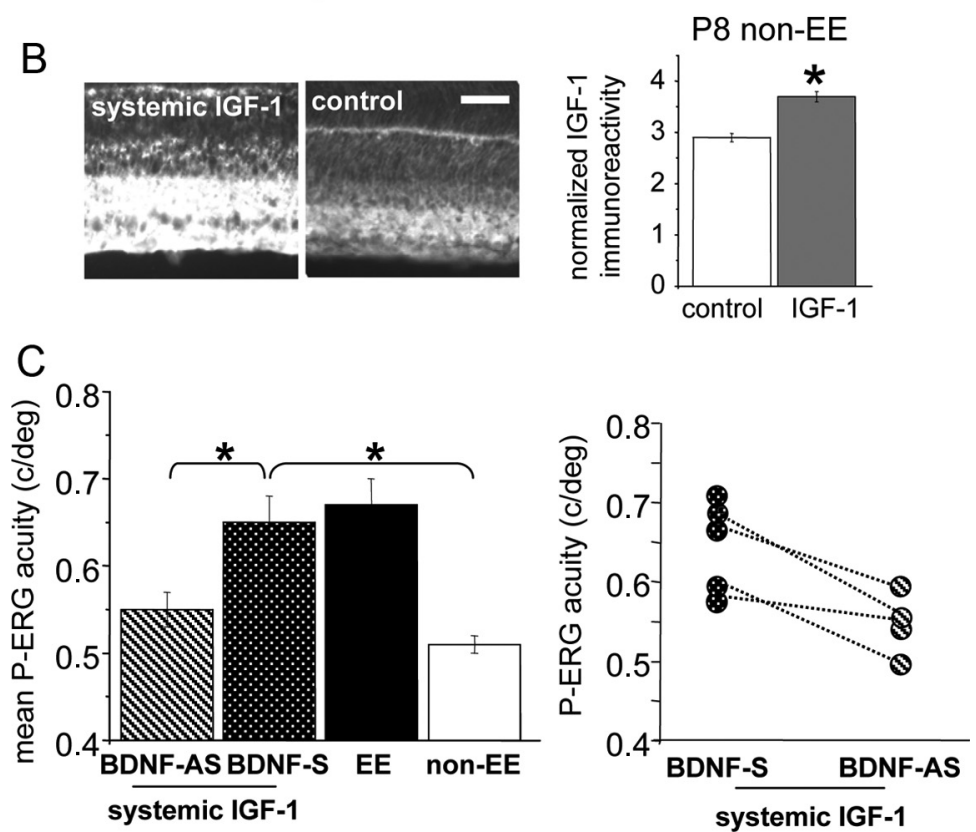

Figure 4. Block of BDNF in the retina prevents the increase of retinal acuity present at P25 in IGF-1-treated rats. $A$, Schematic protocol of the experiment. $\boldsymbol{B}$, Left, Representative images taken from P8 retinas processed for IGF-1 immunoreactivity. Right, Normalized IGF-1 expression in the RGC layer. IGF- 1 levels in the RGC layer of IGF-1 systemically infused non-EE rats $(3.72 \pm 0.24 ; n=4)$ are significantly higher (asterisks) than in control non-EE rats $(2.92 \pm 0.16 ; n=4)(t$ test, $p=0.002)$. Scale bar, $50 \mu \mathrm{m}$. Error bars represent SEM. C, Left, Mean retinal acuity at P25 for IGF-1 systemically injected pups that had been intraocularly injected with BDNF-AS oligonucleotides $(n=4)$ in one eye and with BDNF-Soligonucleotides $(n=5)$ in the other. P-ERG acuity for the eye treated with antisense BDNF oligonucleotides is significantly lower $[0.55 \pm 0.02$ cycles/degree $(\mathrm{c} / \mathrm{deg})]$ than in sense-treated eyes $(0.65 \pm 0.03 \mathrm{c} / \mathrm{deg})$ or untreated eyes of EE animals $(0.67 \pm 0.03 \mathrm{c} / \mathrm{deg} ; n=5)$ and does not differ from the P-ERG acuity of P25 non-EE animals $(0.51 \pm 0.01 \mathrm{c} / \mathrm{deg} ; n=5)$ [one-way ANOVA, $p<0.001$, posthoc Tukey'stest; BDNF-AS-treated eye vs EEeyes, $p=0.009$ (asterisk);BDNF-AS-treated eyes vs BDNF-S-treated eyes, $p=$ 0.032 (asterisk); BDNF-AS-treated eyes vs non-EE eyes, $p=0.572$; BDNF-S-treated eyes vs non-EE eyes, $p<0.05$ (asterisk)]. Error bars represent SEM. Right, Data for single IGF-1 systemically treated animals injected in one eye with BDNF-AS and in the contralateral eye with BDNF-S oligos. In four animals, retinal acuity was determined for both eyes (data points joined by dotted lines); it is evident that for these animals, the acuity of the antisense-treated eye is lower than that for the contralateral eye (paired $t$ test, $p=0.027$ ).

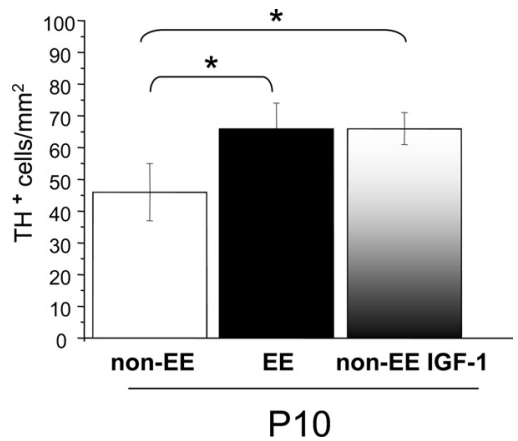

Figure 5. IGF-1 increases the number of amacrine dopaminergic cells. The number of TH-positive amacrine cells is significantly higher in P10 IGF-1-treated non-EE rats than in non-EE untreated animals $\left(67 \pm 5\right.$ vs $46 \pm 3$ cells $\left./ \mathrm{mm}^{2}\right)$. The effect of IGF-1 administration is comparable with that induced by EE: the number of TH-positive amacrine cells of P10 IGF-1-treated non-EE rats did not differ from that of P10 EE rats $\left(66 \pm 3\right.$ cells $/ \mathrm{mm}^{2}$ ) (one-way ANOVA, $p=0.005$, post hoc Tukey's test; non-EE untreated eyes vs IGF-1-treated eyes, $p=0.008$ (asterisk); non-EE untreated eyes vs EE untreated eyes, $p=0.015$ (asterisk); EE untreated eyes vs IGF-1-treated eyes, $p=0.998$ ). Error bars represent SEM.
1998), and BDNF signaling modulates maturation of TH-immunoreactive amacrine cells (Liu et al., 2007). Dopaminergic input has been suggested to contribute to RGC receptive field organization in animals (for review, see Witkovsky, 2004). In addition, in children with phenylketonuria who experienced very high phenylalanine levels in the first postnatal days, and who should therefore have particularly low levels of dopamine in the retina, visual acuity is lower than in normal ones $(\mathrm{Mu}-$ nakata et al., 2004). Furthermore, a reduction in retinal dopamine, as occurs in parkinsonian patients, results in reduced retinal spatial resolution (Peppe et al., 1998).

We therefore tested the hypothesis that the dopaminergic input could be involved in the effects produced by EE on the retinal acuity maturation.

We have assessed the expression of $\mathrm{TH}$ by immunohistochemistry and quantified the number of TH-positive cells in the retina of P10 EE, non-EE, and non-EE animals intraocularly injected with IGF-1. $\mathrm{P} 10$ is the age at which a strong increment in BDNF expression is already evident in EE and IGF-1-treated non-EE rats with respect to non-EE control animals. We found (Fig. 5) that the number of $\mathrm{TH}$ positive amacrine cells was significantly higher in P10 IGF-1-treated non-EE rats than in non-EE untreated animals. The effect of IGF-1 administration is comparable with that induced by EE: the number of TH-positive amacrine cells in P10 IGF1-treated non-EE rats did not differ from that of P10 EE rats, indicating that a comparable change in the development of the dopaminergic network is triggered by enhanced levels of IGF-1/BDNF protein expression or $\mathrm{EE} \mathrm{(} p=0.005$, one-way ANOVA and post hoc Tukey's test; non-EE untreated eyes vs IGF-1-treated eyes, $p=0.008$; non-EE untreated eyes vs EE untreated eyes, $p=$ 0.015; EE untreated eyes $v s$. IGF-1-treated eyes, $p=0.998$ ).

We then analyzed whether the effect of EE on the dopaminergic system contributes to the maturation of retinal acuity.

To answer this question, we decreased $\mathrm{TH}$ expression in the retina of EE rats by injecting antisense oligonucleotides against $\mathrm{TH}$. We first tested whether antisense oligos against $\mathrm{TH}$ reduce $\mathrm{TH}$ expression in the retina of EE rats by means of immunohistochemistry. EE rats were intraocularly injected with TH-AS or TH-MS oligos at P6 and P9 (Fig. 6A) and $24 \mathrm{~h}$ after the last injection the eyes have been removed and processed for $\mathrm{TH}$ immunohistochemistry. We found that the number of TH-positive cells was lower in the retinas of EE animals treated with TH-AS compared with retinas of EE animals treated with TH-MS or retinas of EE untreated animals (Fig. 6B). We cannot say whether the decreased number of TH-positive cells detected in EE rats intraocularly injected with TH-AS is attributable to a reduced expression level of $\mathrm{TH}$ in amacrine cells or to a reduc- 
tion in the number of these cells. Previous experiments using the same TH-AS oligonucleotides used by us would suggest that they do not produce nonspecific effects in terms of neuronal toxicity and/or general disruption of protein synthesis (Skutella et al., 1997). In accordance, the number of TH-positive cells in EE animals treated with TH-AS did not differ from that in non-EE animals $(p=0.001$, one-way ANOVA and post hoc Tukey's test; TH-AS vs EE, $p<0.001$; TH-AS vs TH-MS, $p=$ 0.004 ; TH-AS vs non-EE, $p=0.316$ ).

We then treated a group of EE animals with anti-TH oligonucleotides in one eye (TH-AS) and with TH-MS oligonucleotides as the control in the other eye (THMS) at P6 and P9 and recorded P-ERG at $\mathrm{P} 25$. We found that retinal acuity in the eyes of TH-MS-treated animals did not differ from that of the eyes of EE untreated animals, whereas retinal acuity in EE animals injected with TH-AS was significantly lower than in EE rats and did not differ from that of non-EE rats of the same age $(p=0.001$, one-way ANOVA and post hoc Tukey's test; EE untreated eyes vs TH-AS-treated eyes, $p<0.001$; EE untreated eyes vs TH-MS-treated eyes, $p=$ 0.208 ; TH-AS-treated eyes vs non-EE untreated eyes, $p=0.879$; TH-MS-treated eyes vs TH-AS-treated eyes, $p=0.010$ ) (Fig. $6 C$ ). In five animals, retinal acuity was determined for both eyes (Fig. 6C); it is evident that for all of these animals, the acuity of the TH-AS-treated eye is lower than that for the TH-MS-treated contralateral eye (paired $t$ test, $p=0.026$ ).

Thus, the reduction of retinal $\mathrm{TH}$ blocks the effects of $\mathrm{EE}$ on the retinal functional maturation.

\section{Discussion}

We have provided a direct demonstration that the early postnatal increase in retinal IGF-1 is a key mediator of EE effects on retinal acuity development. IGF-1 action in controlling retinal maturation requires retinal $\mathrm{BDNF}$ and involves the dopaminergic amacrine cell network.

\section{IGF-1 and retinal maturation}

Enrichment during pregnancy increases IGF-1 levels in fetal retina, and this early increase is involved in mediating the acceleration of RGC migration and death caused by EE in the fetus (Sale et al., 2007). Here, we show that a strong increment in retinal IGF-1 expression is evident in EE animals also postnatally, between P1 and P6, and that this increase in retinal IGF-1 in EE animals is necessary and sufficient for EE to produce its effects also on later stages of retinal development.

We have recently shown that exposure to EE accelerates retinal acuity development with respect to the developmental time course found in non-EE animals (Landi et al., 2007a); the final

B
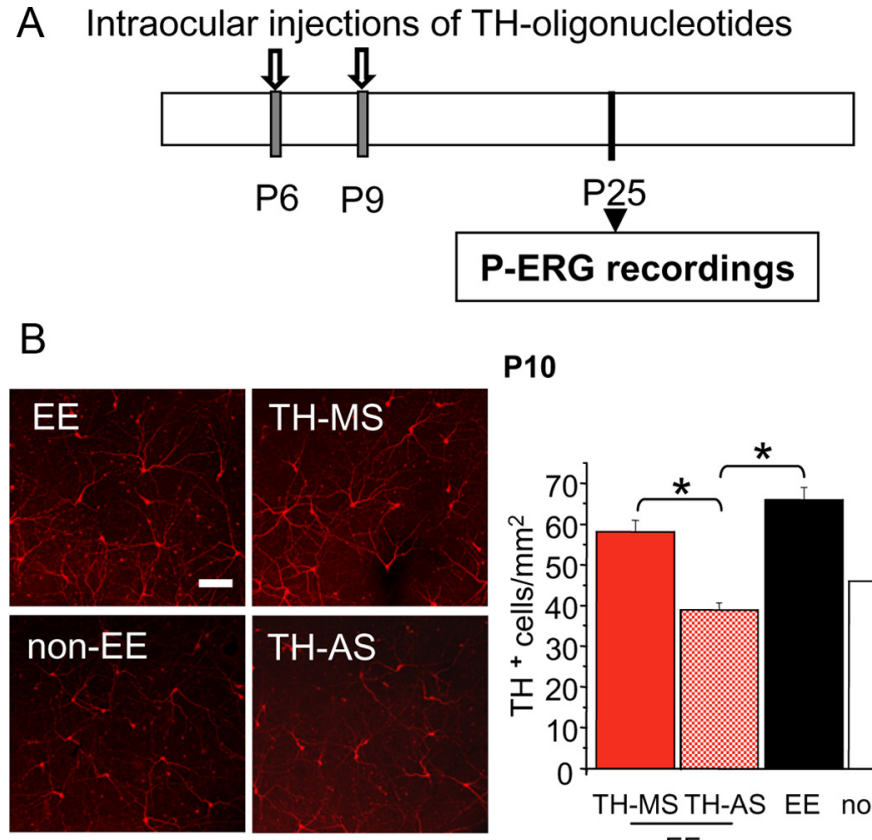

P10
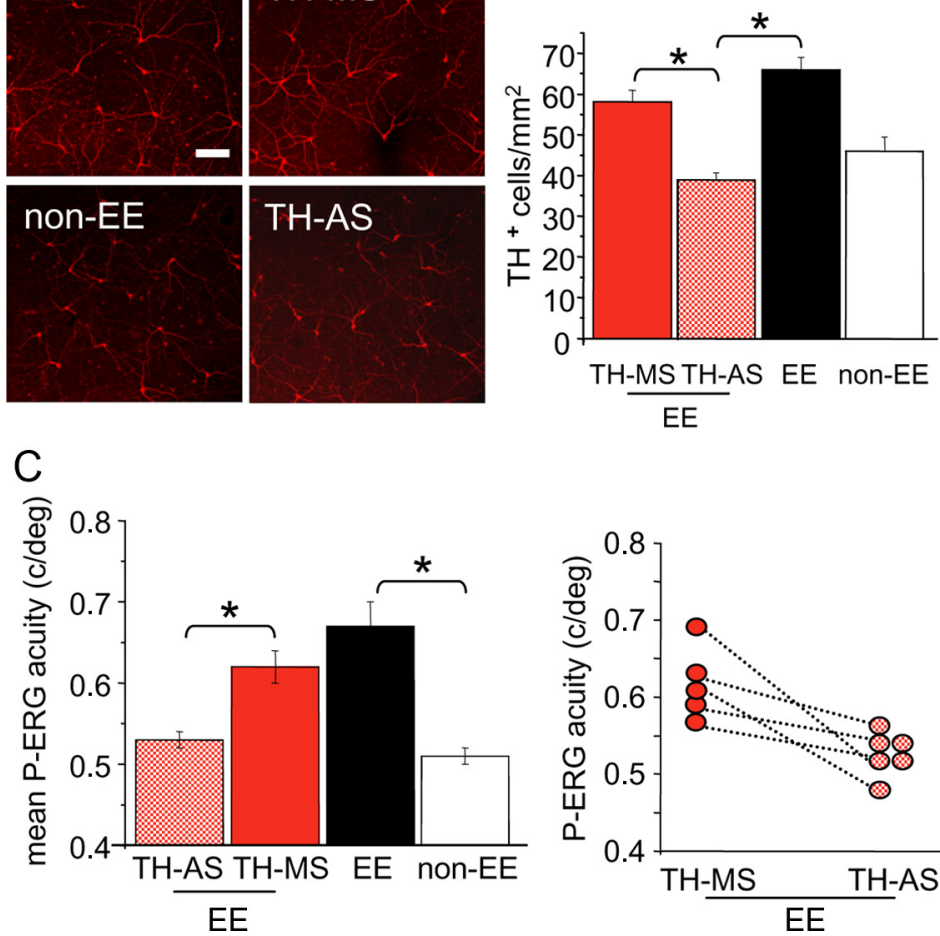

Figure 6. Early blockade of TH prevents the effects of EE on retinal acuity. $\boldsymbol{A}$, Schematic protocol. $\boldsymbol{B}$, Left, Examples of images from P10 whole-mount retinas taken from eyes of EE and non-EE rats or of EE rats intraocularly injected at P6 and P9 with TH-AS or TH-MS oligonucleotides. Right, The number of TH-positive cells is reduced by the treatment with antisense oligonucleotides with respect to the mismatch treatment. In retinas from EE rats injected with TH-AS $(n=4)$, the number of TH-positive cells is significantly lower (asterisks) than in retinas of EE animals treated with TH-MS $(n=4)\left(39 \pm 2 \mathrm{vs} 58 \pm 3 \mathrm{cell} / \mathrm{s} / \mathrm{mm}^{2}\right)$ and in retinas from EE untreated animals ( $66 \pm 3$ cells $/ \mathrm{mm}^{2} ; n=5$ ) but does not differ from that in non-EE retinas ( $46 \pm 3$ cell $/ \mathrm{mm}^{2} ; n=6$ ) (one-way ANOVA, $p=0.001$ ). Scale bar, $100 \mu \mathrm{m}$. Error bars represent SEM. $C$, In a group of six EE animals injected at P6 and P9 with TH-AS in one eye and with TH-MS as a control in the other eye, retinal acuity was assessed with P-ERG at P25. After EE, retinal acuity in TH-MS eyes did not differ from that of EE eyes [0.62 \pm 0.02 vs $0.67 \pm 0.03$ cycles/degree (c/deg)], whereas retinal acuity after EE in TH-AS animals was significantly lower than in EE rats and did not differ from that of non-EE rats of the same age [0.53 \pm 0.01 vs $0.51 \pm 0.01 \mathrm{c} / \mathrm{deg}$; one-way ANOVA, $p=0.001$, post hoc Tukey's test; EE untreated eyes vs EE TH-AS-treated eyes, $p<$ 0.001 (asterisk); EE untreated eyes vs EE TH-MS-treated eyes, $p=0.208$; EE TH-AS-treated eyes vs non-EE untreated eyes, $p=$ was determined for both eyes (data points joined by dotted lines); it is evident that for all of these animals the acuity of the TH-AS-treated eye is lower than that for the TH-MS-treated contralateral eye (paired $t$ test, $p=0.026$ ).

retinal acuity levels do not differ between EE and non-EE rats, suggesting that $\mathrm{EE}$ affects the developmental trajectory rather than the final result of the process of retinal acuity development. The two curves start to differ at P25, when retinal acuity for EE rats first becomes higher than in non-EE rats (Landi et al., 2007a). Here, we show that IGF-1 injections in the eyes of non-EE rat pups between P1 and P7 is sufficient to mimic EE effects in determining a higher retinal acuity at P25. We think that our results would suggest that early IGF-1 treatment triggers a faster increase in retinal acuity such as that after early exposure to EE.

IGF-1 increase in the retina of EE animals is also necessary for EE to affect retinal acuity development, since blockade of IGF-1 
action in EE pups by means of intraocular injections of JB1 prevents EE effects. This is in accordance with results indicating that molecular changes activating the acceleration of retinal maturation are likely influenced by EE precociously, when pups are still immobile and dependent on the dam (Landi et al., 2007a,b); a strong support to this hypothesis is that a paradigm of EE up to $\mathrm{P} 10$ produces the same effects on retinal maturation induced by a prolonged EE, as reported previously by Landi et al. (2007a).

IGF-1 is expressed in the liver, and then is blood circulated (Trejo et al., 2007), but it is also expressed locally in the retina (Bondy, 1991; Lee et al., 1992; Lofqvist et al., 2009). Early exposure of rat pups to EE increases IGF-1 protein levels not only in the retina but also in the cerebellum and maternal milk (Sale et al., 2007). This correlated IGF-1 increase in different brain regions and in the gastric content of EE rat pups would support the idea that EE effects are not specific for the visual system and suggests that the increased retinal presence of IGF-1 in EE animals might derive from a higher level of circulating IGF-1. Tactile stimulation provided by maternal licking behavior, which is increased in EE (Sale et al., 2004), might be responsible for IGF-1 increase. Indeed, in rat pups subjected to massage, we found a higher IGF-1 immunoreactivity in the visual cortex, comparable to that found in EE rat pups, and in the auditory cortex (Guzzetta et al., 2009); in addition, higher contents of blood IGF-1 have been found in human babies subjected to massage (Field et al., 2008; Guzzetta et al., 2009). In massaged babies and rat pups, we found a more rapid increase in visual acuity (Guzzetta et al., 2009). These results support the idea that circulating IGF-1 is an important source of IGF-1 for the CNS in EE animals. However, we cannot exclude a contribution of an increased IGF-1 mRNA expression in the retina of EE pups, since IGF-1 expression in the retina and, in particular, in the RGC layer is well documented (Bondy, 1991; Lee et al., 1992; Lofqvist et al., 2009).

\section{IGF-1 and BDNF}

We have already demonstrated that the increased retinal expression of BDNF induced by EE around P10 is necessary for the effects of EE on retinal acuity and RGC dendritic maturation (Landi et al., 2007a,b). In the adult, BDNF expression is increased both by EE and physical exercise (Carro et al., 2000; Cotman and Berchtold, 2002; Adlard and Cotman, 2004; Klintsova et al., 2004), and this increase is mediated by IGF-1 (Carro et al., 2000). IGF-1 affects the BDNF system to mediate the effects of physical exercise on cognitive processes and synaptic plasticity (Ding et al., 2006). Moreover, IGF-1 promotes neuronal survival and plasticity in primary cultures of cerebrocortical neurons by acting directly on BDNF through ERK1/2 phosphorylation (McCusker et al., 2006). Here, we show for the first time that BDNF is a downstream target of IGF-1 during retinal development and that the early IGF-1 increase found in EE animals is necessary and sufficient for the action of EE on BDNF. Indeed, early IGF-1 injections in the eyes of non-EE pups increase BDNF expression in the RGC layer, whereas blockade of IGF-1 action in EE pups prevents EE effects on BDNF expression. Thus, EE effects on BDNF in the developing retina seem to be mediated by IGF-1.

BDNF is a necessary downstream target of IGF-1 in its action on retinal acuity development: blocking retinal BDNF expression by means of antisense oligonucleotides in non-EE rats treated with systemic IGF-1 injections prevents IGF-1 from affecting retinal acuity development. This is consistent with the crucial role already demonstrated for BDNF in mediating EE effects on retinal development (Landi et al., 2007a,b) and, in particular, with the block of EE effects on retinal acuity development found in EE animals with block of retinal BDNF action. Thus, the early IGF-1 increase determined by EE exposure is unable to trigger the increase in retinal acuity at $\mathrm{P} 25$ if BDNF expression is antagonized. We do not know whether BDNF is the sole target of IGF-1 in regulating retinal acuity development; for instance, several findings provide evidence that IGF-1 and vascular endothelial growth factor (VEGF) act in concert in promoting retinal vascular maturation (Modanlou et al., 2006; Chen and Smith, 2007). However, since BDNF is not dispensable for IGF-1 action on retinal acuity development, one might suggest that other IGF-1 targets either regulate other aspects of retinal development or are cofactors with BDNF in regulating retinal spatial resolution development.

\section{EE, IGF-1, BDNF, and amacrine cell network}

As a target of IGF-1/BDNF control on retinal acuity development of EE animals, we propose a role for the dopaminergic amacrine cell network. Previous studies have suggested that retinal dopamine level affects visual acuity development (Munakata et al., 2004). It is known that dopaminergic amacrine cells express BDNF receptor TrkB (Cellerino and Kohler, 1997), their development is accelerated by BDNF intraocular injections from $\mathrm{P} 8$ to P14 (Cellerino et al., 1998), and BDNF overexpression increases the number of TH-immunoreactive amacrine cells (Liu et al., 2007). In addition, BDNF increases survival of dopaminergic amacrine cells and upregulates TH expression in pathological conditions (Seki et al., 2004; Lee et al., 2005; Loeliger et al., 2008). Potentially, EE and IGF-1, which cause an increase in retinal BDNF, could affect dopaminergic amacrine cells. We have provided direct evidence that this is indeed the case: the number of $\mathrm{TH}$-immunoreactive cells at $\mathrm{P} 10$ is higher in rats exposed to EE and in non-EE rats intraocularly injected with IGF-1 with respect to controls. Thus, dopaminergic amacrine cells are similarly affected by IGF-1 and by exposure to EE; this is consistent with the role for IGF-1 in EE-dependent retinal development. In addition, the results that a reduction in TH expression, obtained by injecting TH-AS oligos in the eye, prevents EE from affecting retinal acuity development suggest that changes in dopamine transmission in EE retinas are necessary for the effects of EE on maturation of retinal acuity.

How could dopamine affect retinal acuity development? Dopamine affects many elements of retinal circuits: it alters the coupling between horizontal cells and between amacrine cells (Piccolino et al., 1984; Hampson et al., 1992), probably via a paracrine release (He et al., 2000) and ultimately affects the center-surround balance of RGCs (Jensen and Daw, 1984, 1986; for review, see Masland, 2001; for review, see Witkovsky, 2004). Spatial tuning of RGCs is very likely to contribute to retinal acuity. We cannot exclude that the spatial tuning of RGCs depends on other retinal GABAergic amacrine subpopulations (Sinclair et al., 2004) and other subtypes of amacrine cells also respond to BDNF during development, as documented by changes in the expression of neuropeptides colocalizing with GABA (Cellerino et al., 1999, 2003). Furthermore, BDNF could act directly on RGCs. Many findings have demonstrated that BDNF shapes dendritic morphology (Purves et al., 1988; McAllister et al., 1995; Schwartz et al., 1997; Xu et al., 2000); interestingly, it has been shown that locally released BDNF can have an autocrine/paracrine action on RGCs, promoting the retraction of RGC dendritic arborization in Xenopus tadpoles (Lom et al., 2002). Recently, we showed that EE promotes, through the action of BDNF, the remodeling of RGC dendritic arborizations accelerating the RGC maturation from monostratified to bistratified, which is a classical feature of retinal 
development (Landi et al., 2007b). Although a correspondence between development of RGC dendritic arborization and maturation of receptive field size, and consequently of retinal acuity, is not immediate (Rusoff and Dubin, 1978), we do not exclude the possibility that a remodeling action of retinal BDNF on RGC dendrites may contribute to retinal acuity maturation.

Nonetheless, our results that reducing TH expression prevents EE from affecting retinal functional development strongly suggest that dopamine is necessary for $\mathrm{EE}$ action on retinal acuity development.

In conclusion, our results are the first demonstration that $\mathrm{EE}$ effects on the maturation of RGC functional properties require a precise molecular signaling mediated by IGF-1 and BDNF and involving the dopaminergic amacrine cell network.

IGF-1, acting through VEGF (Hellstrom et al., 2001, 2003; for review, Chen and Smith, 2007), is critical for retinal vascular development; in particular, low levels of serum IGF-1, often found in low-birth-weight or premature babies and in infants with insufficient nutrient intake, causing disruption of retinal vascularization, appear to correlate with an increased risk of retinopathy of prematurity. Our results emphasize a role for IGF-1 also for neural retinal development, both in the early and the late phases.

\section{References}

Adlard PA, Cotman CW (2004) Voluntary exercise protects against stressinduced decreases in brain-derived neurotrophic factor protein expression. Neuroscience 124:985-992.

Berardi N, Domenici L, Gravina A, Maffei L (1990) Pattern ERG in rats following section of the optic nerve. Exp Brain Res 79:539-546.

Bondy CA (1991) Transient IGF-I gene expression during the maturation of functionally related central projection neurons. J Neurosci 11:3442-3455.

Cancedda L, Putignano E, Sale A, Viegi A, Berardi N, Maffei L (2004) Acceleration of visual system development by environmental enrichment. J Neurosci 24:4840-4848.

Carro E, Nunez A, Busiguina S, Torres-Aleman I (2000) Circulating insulinlike growth factor I mediates effects of exercise on the brain. J Neurosci 20:2926-2933.

Cellerino A, Kohler K (1997) Brain-derived neurotrophic factor/neurotrophin-4 receptor TrkB is localized on ganglion cells and dopaminergic amacrine cells in the vertebrate retina. J Comp Neurol 386:149-160.

Cellerino A, Pinzon-Duarte G, Carroll P, Kohler K (1998) Brain-derived neurotrophic factor modulates the development of the dopaminergic network in the rodent retina. J Neurosci 18:3351-3362.

Cellerino A, Arango-Gonzalez BA, Kohler K (1999) Effects of brain-derived neurotrophic factor on the development of NADPH-diaphorase/nitric oxide synthase-positive amacrine cells in the rodent retina. Eur J Neurosci 11:2824-2834.

Cellerino A, Arango-Gonzalez B, Pinzon-Duarte G, Kohler K (2003) Brainderived neurotrophic factor regulates expression of vasoactive intestinal polypeptide in retinal amacrine cells. J Comp Neurol 467:97-104.

Chen J, Smith LE (2007) Retinopathy of prematurity. Angiogenesis 10:133-140.

Ciucci F, Putignano E, Baroncelli L, Landi S, Berardi N, Maffei L (2007) Insulin-like growth factor 1 (IGF-1) mediates the effects of enriched environment (EE) on visual cortical development. PLoS ONE 2:e475.

Cotman CW, Berchtold NC (2002) Exercise: a behavioral intervention to enhance brain health and plasticity. Trends Neurosci 25:295-301.

Ding Q, Vaynman S, Akhavan M, Ying Z, Gomez-Pinilla F (2006) Insulin-like growth factor I interfaces with brain-derived neurotrophic factor-mediated synaptic plasticity to modulate aspects of exercise-induced cognitive function. Neuroscience 140:823-833.

Domenici L, Berardi N, Carmignoto G, Vantini G, Maffei L (1991) Nerve growth factor prevents the amblyopic effects of monocular deprivation. Proc Natl Acad Sci U S A 88:8811-8815.

Elmlinger MW, Sanatani MS, Bell M, Dannecker GE, Ranke MB (1998) Elevated insulin-like growth factor (IGF) binding protein (IGFBP)-2 and IGFBP-4 expression of leukemic T-cells is affected by autocrine/paracrine
IGF-II action but not by IGF type I receptor expression. Eur J Endocrinol 138:337-343.

Etgen AM, Acosta-Martinez M (2003) Participation of growth factor signal transduction pathways in estradiol facilitation of female reproductive behavior. Endocrinology 144:3828-3835.

Fernandez AM, Garcia-Estrada J, Garcia-Segura LM, Torres-Aleman I (1997) Insulin-like growth factor I modulates c-Fos induction and astrocytosis in response to neurotoxic insult. Neuroscience 76:117-122.

Field T, Diego M, Hernandez-Reif M, Dieter JN, Kumar AM, Schanberg S, Kuhn C (2008) Insulin and insulin-like growth factor-1 increased in preterm neonates following massage therapy. J Dev Behav Pediatr 29:463-466.

Guzzetta A, Baldini S, Bancale A, Baroncelli L, Ciucci F, Ghirri P, Putignano E, Sale A, Viegi A, Berardi N, Boldrini A, Cioni G, Maffei L (2009) Massage accelerates brain development and the maturation of visual function. J Neurosci, 29:6042-6051.

Hampson EC, Vaney DI, Weiler R (1992) Dopaminergic modulation of gap junction permeability between amacrine cells in mammalian retina. J Neurosci 12:4911-4922.

Hellstrom A, Perruzzi C, Ju M, Engstrom E, Hard AL, Liu JL, AlbertssonWikland K, Carlsson B, Niklasson A, Sjodell L, LeRoith D, Senger DR, Smith LE (2001) Low IGF-I suppresses VEGF-survival signaling in retinal endothelial cells: direct correlation with clinical retinopathy of prematurity. Proc Natl Acad Sci U S A 98:5804-5808.

Hellstrom A, Engstrom E, Hard AL, Albertsson-Wikland K, Carlsson B, Niklasson A, Lofqvist C, Svensson E, Holm S, Ewald U, Holmstrom G, Smith LE (2003) Postnatal serum insulin-like growth factor I deficiency is associated with retinopathy of prematurity and other complications of premature birth. Pediatrics 112:1016-1020.

He S, Weiler R, Vaney DI (2000) Endogenous dopaminergic regulation of horizontal cell coupling in the mammalian retina. J Comp Neurol 418:33-40.

Humbel RE (1990) Insulin-like growth factors I and II. Eur J Biochem 190:445-462.

Jensen RJ, Daw NW (1984) Effects of dopamine antagonists on receptive fields of brisk cells and directionally selective cells in the rabbit retina. J Neurosci 4:2972-2985.

Jensen RJ, Daw NW (1986) Effects of dopamine and its agonists and antagonists on the receptive field properties of ganglion cells in the rabbit retina. Neuroscience 17:837-855.

Klintsova AY, Dickson E, Yoshida R, Greenough WT (2004) Altered expression of BDNF and its high-affinity receptor TrkB in response to complex motor learning and moderate exercise. Brain Res 1028:92-104.

Landi S, Sale A, Berardi N, Viegi A, Maffei L, Cenni MC (2007a) Retinal functional development is sensitive to environmental enrichment: a role for BDNF. FASEB J 21:130-139.

Landi S, Cenni MC, Maffei L, Berardi N (2007b) Environmental enrichment effects on development of retinal ganglion cell dendritic stratification require retinal BDNF. PLoS ONE 2:e346.

Lee EJ, Song MC, Kim HJ, Lim EJ, Kim IB, Oh SJ, Moon JI, Chun MH (2005) Brain-derived neurotrophic factor modulates the dopaminergic network in the rat retina after axotomy. Cell Tissue Res 322:191-199.

Lee WH, Javedan S, Bondy CA (1992) Coordinate expression of insulin-like growth factor system components by neurons and neuroglia during retinal and cerebellar development. J Neurosci 12:4737-4744.

Liu X, Grishanin RN, Tolwani RJ, Renteria RC, Xu B, Reichardt LF, Copenhagen DR (2007) Brain-derived neurotrophic factor and TrkB modulate visual experience-dependent refinement of neuronal pathways in retina. J Neurosci 27:7256-7267.

Loeliger MM, Briscoe T, Rees SM (2008) BDNF increases survival of retinal dopaminergic neurons after prenatal compromise. Invest Ophthalmol Vis Sci 49:1282-1289.

Lofqvist C, Willett KL, Aspegren O, Smith AC, Aderman CM, Connor KM, Chen J, Hellstrom A, Smith LE (2009) Quantification and localization of the IGF/insulin system expression in retinal blood vessels and neurons during oxygen-induced retinopathy in mice. Invest Ophthalmol Vis Sci 50:1831-1837.

Lom B, Cogen J, Sanchez AL, Vu T, Cohen-Cory S (2002) Local and targetderived brain-derived neurotrophic factor exert opposing effects on the dendritic arborization of retinal ganglion cells in vivo. J Neurosci 22: 7639-7649.

Mandolesi G, Menna E, Harauzov A, von Bartheld CS, Caleo M, Maffei L 
(2005) A role for retinal brain-derived neurotrophic factor in ocular dominance plasticity. Curr Biol 15:2119-2124.

Masland RH (2001) The fundamental plan of the retina. Nat Neurosci 4:877-886.

McAllister AK, Lo DC, Katz LC (1995) Neurotrophins regulate dendritic growth in developing visual cortex. Neuron 15:791-803.

McCusker RH, McCrea K, Zunich S, Dantzer R, Broussard SR, Johnson RW, Kelley KW (2006) Insulin-like growth factor-I enhances the biological activity of brain-derived neurotrophic factor on cerebrocortical neurons. J Neuroimmunol 179:186-190.

Menna E, Cenni MC, Naska S, Maffei L (2003) The anterogradely transported BDNF promotes retinal axon remodeling during eye specific segregation within the LGN. Mol Cell Neurosci 24:972-983.

Modanlou HD, Gharraee Z, Hasan J, Waltzman J, Nageotte S, Beharry KD (2006) Ontogeny of VEGF, IGF-I, and GH in neonatal rat serum, vitreous fluid, and retina from birth to weaning. Invest Ophthalmol Vis Sci $47: 738-744$.

Munakata Y, Casey BJ, Diamond A (2004) Developmental cognitive neuroscience: progress and potential. Trends Cogn Sci 8:122-128.

Peppe A, Stanzione P, Pierantozzi M, Semprini R, Bassi A, Santilli AM, Formisano R, Piccolino M, Bernardi G (1998) Does pattern electroretinogram spatial tuning alteration in Parkinson's disease depend on motor disturbances or retinal dopaminergic loss? Electroencephalogr Clin Neurophysiol 106:374-382.

Piccolino M, Neyton J, Gerschenfeld HM (1984) Decrease of gap junction permeability induced by dopamine and cyclic adenosine $3^{\prime}: 5^{\prime}$-monophosphate in horizontal cells of turtle retina. J Neurosci 4:2477-2488.

Pozios KC, Ding J, Degger B, Upton Z, Duan C (2001) IGFs stimulate zebrafish cell proliferation by activating MAP kinase and PI3-kinase-signaling pathways. Am J Physiol Regul Integr Comp Physiol 280:R1230-R1239.

Probst JC, Skutella T (1996) The G-tetrad in antisense targeting. Trends Genet 12:290-291

Purves D, Snider WD, Voyvodic JT (1988) Trophic regulation of nerve cell morphology and innervation in the autonomic nervous system. Nature 336:123-128.

Rodrigues M, Waldbillig RJ, Rajagopalan S, Hackett J, LeRoith D, Chader GJ (1988) Retinal insulin receptors: localization using a polyclonal antiinsulin receptor antibody. Brain Res 443:389-394.

Rossi FM, Pizzorusso T, Porciatti V, Marrubio LM, Maffei L, Changeux JP (2001) Requirement of the nicotinic acetylcholine receptor beta 2 subunit for the anatomical and functional development of the visual system. Proc Natl Acad Sci U S A 98:6453-6458.

Sale A, Putignano E, Cancedda L, Landi S, Citrulli F, Berardi N, Maffei L (2004) Enriched environment and acceleration of visual system development. Neuropharmacology 47:649-660.

Sale A, Cenni MC, Ciucci F, Putignano E, Chierzi S, Maffei L (2007) Mater- nal enrichment during pregnancy accelerates retinal development of the fetus. PLoS ONE 2:e1160.

Schwartz PM, Borghesani PR, Levy RL, Pomeroy SL, Segal RA (1997) Abnormal cerebellar development and foliation in BDNF- - - mice reveals a role for neurotrophins in CNS patterning. Neuron 19:269-281.

Seki M, Tanaka T, Nawa H, Usui T, Fukuchi T, Ikeda K, Abe H, Takei N (2004) Involvement of brain-derived neurotrophic factor in early retinal neuropathy of streptozotocin-induced diabetes in rats: therapeutic potential of brain-derived neurotrophic factor for dopaminergic amacrine cells. Diabetes 53:2412-2419.

Sinclair JR, Jacobs AL, Nirenberg S (2004) Selective ablation of a class of amacrine cells alters spatial processing in the retina. J Neurosci 24:1459-1467.

Skutella T, Schwarting RK, Huston JP, Sillaber I, Probst JC, Holsboer F, Spanagel R (1997) Infusions of tyrosine hydroxylase antisense oligodeoxynucleotide into substantia nigra of the rat: effects on tyrosine hydroxylase mRNA and protein content, striatal dopamine release and behaviour. Eur J Neurosci 9:210-220.

Stewart CE, Rotwein P (1996) Growth, differentiation, and survival: multiple physiological functions for insulin-like growth factors. Physiol Rev 76:1005-1026.

Thongsong B, Bonkobara M, Matsumoto M, Jang JS, Matsuki N, Inaba M, Ono K (2002) Effects of insulin-like growth factor-I on maternal and foetal plasma amino acid levels in pregnant rats. J Vet Med Sci 64:859-861.

Trejo JL, Carro E, Torres-Aleman I (2001) Circulating insulin-like growth factor I mediates exercise-induced increases in the number of new neurons in the adult hippocampus. J Neurosci 21:1628-1634.

Trejo JL, Piriz J, Llorens-Martin MV, Fernandez AM, Bolós M, LeRoith D, Nuñez A, Torres-Aleman I. (2007) Central actions of liver-derived insulin-like growth factor I underlying its pro-cognitive effects. Mol Psychiatry 12:1118-1128.

Waldbillig RJ, Fletcher RT, Somers RL, Chader GJ (1988) IGF-I receptors in the bovine neural retina: structure, kinase activity and comparison with retinal insulin receptors. Exp Eye Res 47:587-607.

Witkovsky P (2004) Dopamine and retinal function. Doc Ophthalmol 108:17-40.

Woodall SM, Breier BH, Johnston BM, Bassett NS, Barnard R, Gluckman PD (1999) Administration of growth hormone or IGF-I to pregnant rats on a reduced diet throughout pregnancy does not prevent fetal intrauterine growth retardation and elevated blood pressure in adult offspring. J Endocrinol 163:69-77.

Xu B, Gottschalk W, Chow A, Wilson RI, Schnell E, Zang K, Wang D, Nicoll RA, Lu B, Reichardt LF (2000) The role of brain-derived neurotrophic factor receptors in the mature hippocampus: modulation of long-term potentiation through a presynaptic mechanism involving TrkB. J Neurosci 20:6888-6897. 